\title{
Design of Sail-Assisted Unmanned Surface Vehicle Intelligent Control System
}

\author{
Yong Ma, ${ }^{1}$ Yujiao Zhao, ${ }^{1,2}$ Jiantao Diao, ${ }^{2}$ Langxiong Gan, ${ }^{1}$ \\ Huaxiong Bi, ${ }^{1}$ and Jingming $\mathrm{Zhao}^{3}$ \\ ${ }^{1}$ School of Navigation, Hubei Key Laboratory of Inland Shipping Technology, Wuhan University of Technology, Wuhan 430063, China \\ ${ }^{2}$ School of Computer Science and Technology, Wuhan University of Technology, Wuhan 430063, China \\ ${ }^{3}$ School of Energy and Power Engineering, Wuhan University of Technology, Wuhan 430063, China \\ Correspondence should be addressed to Yong Ma; myongdl@whut.edu.cn
}

Received 18 August 2016; Revised 6 November 2016; Accepted 7 November 2016

Academic Editor: Guillermo Botella-Juan

Copyright (C) 2016 Yong Ma et al. This is an open access article distributed under the Creative Commons Attribution License, which permits unrestricted use, distribution, and reproduction in any medium, provided the original work is properly cited.

\begin{abstract}
To achieve the wind sail-assisted function of the unmanned surface vehicle (USV), this work focuses on the design problems of the sail-assisted USV intelligent control systems (SUICS) and illustrates the implementation process of the SUICS. The SUICS consists of the communication system, the sensor system, the PC platform, and the lower machine platform. To make full use of the wind energy, in the SUICS, we propose the sail angle of attack automatic adjustment (Sail_4A) algorithm and present the realization flow for each subsystem of the SUICS. By using the test boat, the design and implementation of the SUICS are fulfilled systematically. Experiments verify the performance and effectiveness of our SUICS. The SUICS enhances the intelligent utility of sustainable wind energy for the sail-assisted USV significantly and plays a vital role in shipping energy-saving emission reduction requirements issued by International Maritime Organization (IMO).
\end{abstract}

\section{Introduction}

Featured by large-scale reserves, wide distribution, and little pollution, as one of the popular sustainable energy [1-3], wind energy has been extensively used in the shipping industry. By using the wind energy, numerous studies paid attention to the vessels equipped with the sail. Generally, the present studies mainly emphasize on the aerodynamic performance $[4,5]$ and navigation strategy [6-8] for the sailboat and the sail-assisted vessels. Little attention has stressed the sail-assisted control system for the vessels. Restricted to the petrochemical energy crisis and environmental pollution, International Maritime Organization (IMO) has put forward quite strict requirements for shipping energy conservation and emission reduction. Wind energy would do great deeds for the sail-assisted vessels by way of the effective solutions. It is sane to ascertain that, with the effective control system, the wind energy utilization of the sail-assisted vessel can be achieved with ease.

Meanwhile, the unmanned surface vehicle (USV) has been adopted in the short-range maritime activities including the military and the civil domains. The USV would bring the positive impact on the shipping industry by virtue of its autonomy and flexibility. However, owing to the limited storage space and load capacity, the USV could not facility the large-scale engine or the battery apparatuses that inhibit the navigation time and distance of the USV dramatically. Following that, if the USV is equipped with the sail, then the energy consumption can be lowered down considerably and the navigation distance can be extended naturally. Consequently, the sail-assisted USV intelligent control systems (SUICS) is proposed to exploit sustainable wind energy.

Our SUICS consists of the design and the implementation phases. During the SUICS design phase, the modules including the communication system, the sensor system, the PC platform, and the lower machine platform are detailed sequentially. Within the implementation phase, by using the test boat, the implement flows related to the communication system, the sensor system, and the drive system are expounded, and then each module is integrated into our SUICS. 
Generous types of sails have been applied in the sailassisted vessels. From the perspective of appearance, the sails can be classified into the jib and square sails [5]. And the rectangular sail, the broad top sail, and parallel quadrilateral sail all belong to the square sail. When it comes to the material, the sail can be grouped into the soft sail and hard sail [9]. In terms of the cross-sectional shape, the sail can be divided into the wing sail, the laminar flow sail, the lie back sail, and the circular arc sail. Based on numerous tunnel experiment analyses, it is demonstrated that the circular arc sail is superior to other sails in aspects of the best aerodynamic performance, the simpler operation, and production process [10].

Wind power is one environmentally friendly energy that can be used a substitute for the drying up fossil fuel [4]. Generous researches have investigated the sail-assisted navigation techniques, including the behavior of yacht soft sails [11], fluid-structure interaction issues [12], and unsteady aerodynamic phenomena [13]. In [14], a kite modelling approach into 6 degrees of freedom sailboat dynamic simulator was fulfilled; following that the performances between the kite and classical rig sailing can be evaluated. From the perspective of dynamic simulation, [14] stressed kite propulsion performance from upwind and downwind with one designed keel boat. Most of the current studies [1114] concentrated on the soft sail structures of yachts. To promote the wind energy usage, taking into account the shipping industry requirements, it is vital to develop the hard sail-assisted vessels. Thereupon, the circular arc hard sail is adopted to carry out the SUICS studies.

With the aid of the SUICS, the accurate data related to the wind can be displayed, collected, and processed for the usage of the vessel decision making in real time, and then by using the sail automatic control system the correct actions could be instructed promptly. The SUICS aims at using the wind energy scientifically and intelligently and offering the potential solution to cut down the energy expenditure of the shipping industry. By using the SUICS, the navigation distance of the USV can be extended benefits from the effective use of wind energy. No doubt that, with the embedded SUICS, the energy consumption of the vessels with the assisted sail can be reduced [15].

The contributions of this work are as follows: (1) Design and implement the SUICS to fulfill the effective usage of the sustainable wind energy for the sail-assisted USV; (2) to intelligently control the sail angle of the attack, we propose the automatic adjustment algorithm for the sail automatic control system of the SUICS; (3) to fulfill the human-machine interface friendly, we design the PC platform software for the SUICS; and (4) our designed data frame format is characterized by the quite low transmission error rate and fit for the compact control system and is useful to the communication module of the remote control system.

This paper is organized as follows. Section 2 demonstrates the module design work of the SUICS, including the communication system, the sensor system, the sail automatic control system, the PC platform, and the low machine platform. Then, Section 3 elaborates the implement processes of the

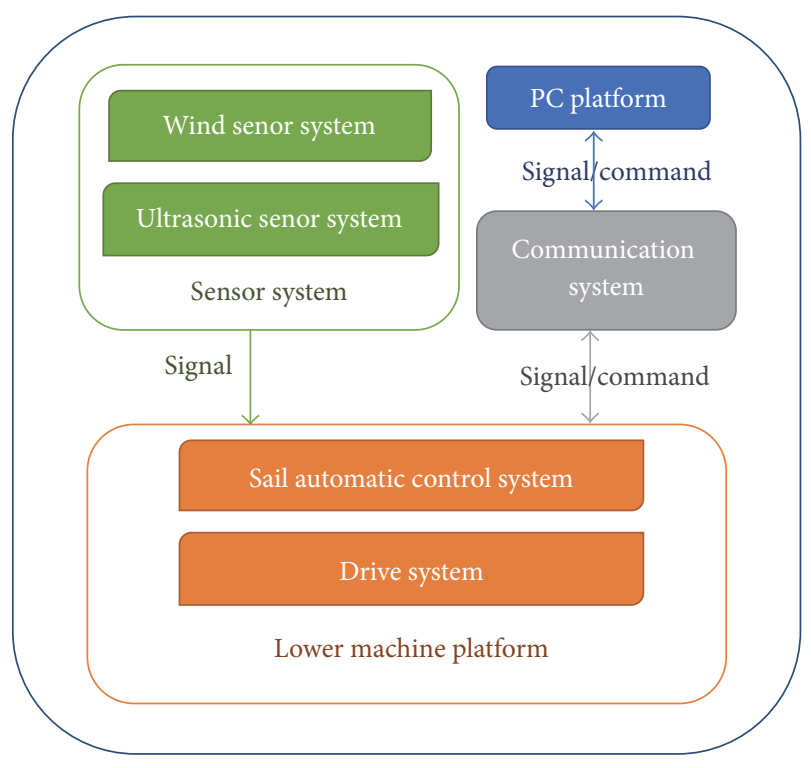

FIgure 1: Logic diagram of the SUICS.

SUICS. In Section 4, experiments verify the effectiveness of the SUCIS. Finally, Section 5 concludes this paper.

\section{Design of the Sail-Assisted USV Intelligent Control Systems}

The communication system, the sensor system, the PC platform, and the lower machine platform compose the SUICS. Figure 1 shows the logic diagram of the SUICS. The sensor system gathers the real time data, including the wind direction and speed, and the environment data; then by way of the serial port communication, the data are transferred to the Single Chip Microcomputer (SCM); following that the SCM adjusts the sail angle of attack automatically by using the data with the proposed sail angle of attack automatic adjustment algorithm. Simultaneously, the data are sent to the PC platform through the communication system; the PC platform would echo data to the user. By using the humanmachine interface, the user could operate the sail through commands directly, the commands are encapsulated in a frame, then the frame will be sent to the lower machine platform, and the lower machine platform parses the frame and executes corresponding actions.

By using the SUICS, the sail angle of attack can be adjusted adaptively; then the sustainable wind energy can be exploited to the maximum. With the aid of the SUICS, the activity domain and time of the sail-assisted USV can be strengthened benefiting from the energy offered by the sustainable wind. Figure 2 depicts the system structure diagram of the SUICS; the embedded programming method is introduced to attain the digital precise control of the sail-assisted USV. The design details of the communication system, the sensor system, the PC platform, and the lower machine platform are sequentially illustrated as shown in Figure 2. 


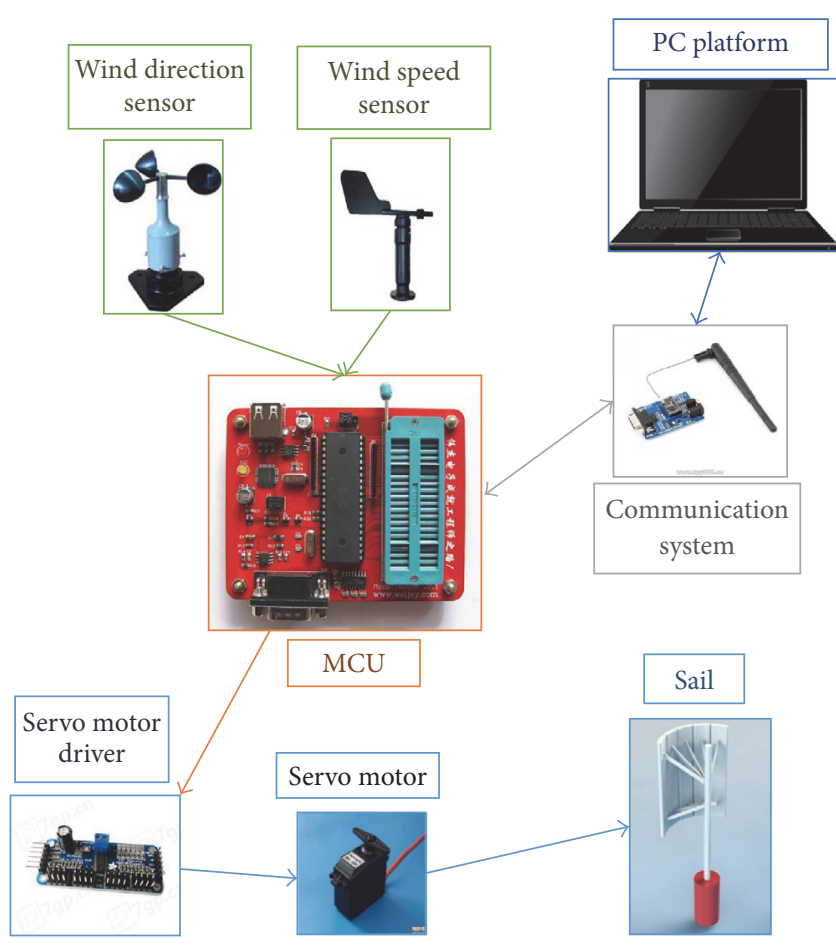

FIGURE 2: System structure diagram of the SUICS.

Excellent works have paid attention to the system architectures from floating point to fixed point [16-19], including analysis of mean-square-error for fixed point FFT units [16], efficient implementation of a spectrum analyzer for fixed point architectures [17], quantization analysis and enhancement of a VLSI gradient-based motion estimation architecture [18], and presentation of robust bioinspired architecture for optical flow computation [19]. When comes to our SUICS, as shown in Figure 2, the range of wind speed sensor is from $0.4 \mathrm{~m} / \mathrm{s}$ to $45.0 \mathrm{~m} / \mathrm{s}$ with $0.1 \mathrm{~m} / \mathrm{s}$ resolution, the resolution of wind direction sensor is $1^{\circ}$, the data acquisition cycle is $3 \mathrm{~s}$, the communication model transfer rate is $150 \mathrm{Mbps}$, and the communication distance is $120 \mathrm{~m}$.

2.1. Communication System. Within the communication system, the network formation and the data frame based on the TCP/IP protocol are stressed.

2.1.1. Communication Network Formation. Generally, with a wireless network card and an AP set, the wireless network can be established as one router. After the PC platform connects to above Wi-Fi module, the PC can interact with the low machine platform placed on the USV in real time [20]. In the communication protocol, the communication module services for the control system, and they belong to data link layer and the application layer, respectively. With respect to the control system, the communication module is transparent and independent; those identities contribute to the convenience to the data process and the maintenance of the platforms [21].
TABLE 1: Data frame format.

\begin{tabular}{lcccc}
\hline $\begin{array}{l}\text { Frame } \\
\text { head }\end{array}$ & $\begin{array}{c}\text { Subsystem } \\
\text { number }\end{array}$ & $\begin{array}{c}\text { Equipment } \\
\text { number }\end{array}$ & Command/data & $\begin{array}{c}\text { Tail } \\
\text { frame }\end{array}$ \\
\hline
\end{tabular}

2.1.2. Data Frame Based on TCP/IP Protocol. Our communication system complies with the TCP/IP protocol [22]. As presented in Table 1, we propose one compacted streamlined data frame format for the small-scale control system. The shorter the length of the data frame format, the faster the data transmission speed. By using our data frame format, the system response speed can be improved and the data conflict can be reduced. When it comes to the data frame solution, the procedure would take the first judgment of the value of the first two bits of the data frame and then process the data of the small-scale control system with one effective data frame solution.

2.2. Data Processing for the Sensor System. During the navigation process, to make sound decisions, the sail-assisted USV should acquire the data related to the wind energy and the environment around itself in real time. By using the wind speed sensor and the wind direction sensor, the raw data are obtained [23]. The wind speed equals $1 / 71$ of its initial frequency signal, and the wind direction data also can be reached after being processed by digital filter. Then, the processed data are delivered to the sail controller placed on the lower machine platform simultaneously. With above data, the sail controller could execute certain actions on the sail and send the data to the PC platform for display.

Owing to the limited space and load capacity of the USV, to detect the environment, including the obstacles around the USV, the radar is not a viable option definitely. Therefore, the portable ultrasonic ranging sensor is adopted to overcome above limitation. We place three ultrasonic ranging sensors in the bow, the port side, and the starboard side successively. Furthermore, to expand the detection width, under each sensor, we install the microservos to steer the sensors spin. Consequently, the detection ability of the ultrasonic ranging sensor is strengthened, and the collected data are fed back to the upper machine platform for decision making.

2.3. Design of the PC Platform Software. The sail-assisted USV has combined the semiautonomous model and the remote control model. Thus, the human-machine interface [24] plays a great role between the PC operator and the subsystems embedded in the lower machine platform. By using the human-machine interface, the operator could acquire the dynamic information around the sail-assisted USV and execute commands on certain equipment with ease. Consequently, the PC platform should satisfy the criteria, including the friendly human-machine interface; the multifunctional applications contribute to access to the dynamic information related to the sail-assisted USV. Taken above criteria into account, within the design process, the QT platform [25] is applied to build the PC platform, and the TCP/IP protocol [26] is adopted to guarantee the quality and reliability of the communication. 


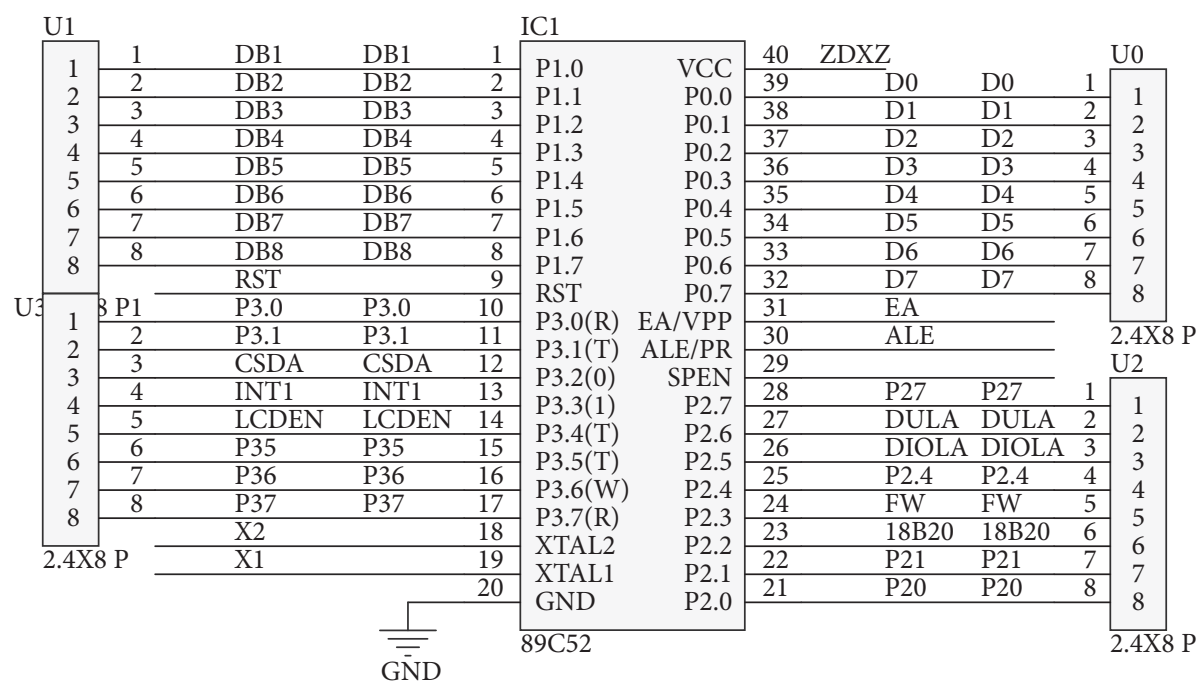

FIgURE 3: The pin of the SCM.

\subsection{Design of the Lower Machine Platform}

2.4.1. Brief Introduction of the Lower Machine Platform. The lower machine platform mainly consists of the sail automatic control system and the drive system and executes the information related to the control of the sail and the motion of the USV; it is the core part of the SUICS on account of the safety and performance of the sail-assisted USV. The distributed processing technology is used to integrate multiple 51 Single Chip Micyoco (SCM) that each SCM dedicates to one special function and actualize the parallel process ability of the systems embedded in the lower machine platform [27]. Following that, the information process speed and accuracy of the lower machine platform can be enhanced, benefiting from the adopted design technologies. Figure 3 shows the pin of the SCM.

In Figure 3, P0 port is a set of eight-bit open drain bidirectional I/O port, and it is an address/data bus multiplexer port. $\mathrm{P} 1$ port is an eight-bit I/O port with internal pullups. During flash programming and program verification, $\mathrm{P} 1$ receives a low eight-bit address, while $\mathrm{P} 2$ receives a high-bit address and other control data. P3 port is an eight-bit I/O port with internal pullups. In addition to as a general $\mathrm{I} / \mathrm{O}$ port, P3.0(RXD) is a serial port to input, P3.1(TXD) is a serial port to output, P3.2(INT0) is the external interrupt zero port, P3.3(INT1) is the external interrupt one port, P3.4(T0) is timer/counter zero, P3.5(T1) is timer/counter one, P3.6(WR) is the external data memory write strobe line, and P3.7(RD) is the external data memory read strobe line.

Design of the sail automatic control system is stressed in the lower machine platform. To design the high-quality sail automatic control system, it is necessary to achieve the optimum sail angle of attack and then by using the effective algorithm to fulfill the automatic adjustment for the sail angle of attack. Following that, we illustrate the procedure of how to obtain the optimum sail angle of attack and our proposed sail angle of attack automatic adjustment algorithm.

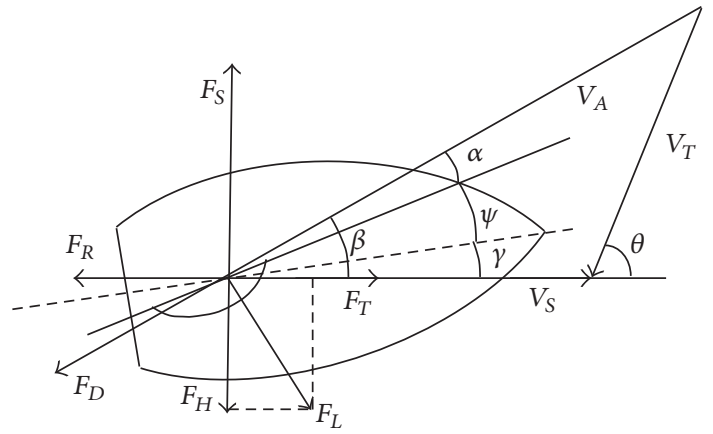

FIgURE 4: Force analysis of the sail.

2.4.2. Procedure to Obtain the Optimum Angle of Attack. Figure 4 shows the force analysis of the sail. Suppose $V_{S}, V_{T}, \theta$, $\gamma, V_{A}, \beta, \alpha$, and $\psi$ represent the speed of the UVS, actual wind speed, the actual wind direction angle between $V_{S}$ and $V_{T}$, the drift angle between $V_{S}$ and the longitudinal axis of the USV, relative wind speed, relative wind direction angle between $V_{A}$ and $V_{S}$, the sail angle of attack, and the turning angle of the sail between the longitudinal axis of the USV and the sailing string, respectively.

When the wind is acting on the sails, the sail will generate a lift force perpendicular to the relative wind speed $F_{L}$ and a resistance along the relative wind speed $F_{D}[28,29]$. $F_{T}$ denotes the thrust parallel to the course of the vessel generated by the sail, and $F_{H}$ denotes the transverse force perpendicular to the direction of the USV. Then, according to the geometrical relationship, $F_{T}$ and $F_{H}$ can be

$$
\begin{aligned}
& F_{T}=F_{L} \sin \beta-F_{D} \cos \beta \\
& F_{H}=F_{L} \cos \beta+F_{D} \sin \beta .
\end{aligned}
$$


After nondimensional process,

$$
\begin{aligned}
& C_{X}=C_{L} \sin \beta-C_{D} \cos \beta \\
& C_{Y}=C_{L} \cos \beta+C_{D} \sin \beta .
\end{aligned}
$$

The lift coefficient, drag coefficient, thrust coefficient, and cornering ratio are sequentially symbolized as $C_{L}, C_{D}$, $C_{X}$, and $C_{Y}$. Suppose $\rho, V, S, \nu, \lambda$, and $\alpha_{\text {opt }}$ stand for air density, wind speed, sail projected area, camber ratio of sail, ratio of sail, and the optimum angle of attack in sequence. According to the relationship between the drag coefficient and lift coefficient with the angle of attack, aspect ratio, and camber ratio, the following functions can be reached:

$$
\begin{aligned}
& C_{L}=f(\alpha, \lambda, \nu) \\
& C_{D}=g(\alpha, \lambda, \nu) \\
& C_{L}=\frac{F_{L}}{(1 / 2) \rho V^{2} S} \\
& C_{D}=\frac{F_{D}}{(1 / 2) \rho V^{2} S} \\
& C_{X}=\frac{F_{T}}{(1 / 2) \rho V^{2} S} \\
& C_{Y}=\frac{F_{D}}{(1 / 2) \rho V^{2} S} .
\end{aligned}
$$

To make full use of sustainable wind energy, it is essential to obtain the maximum thrust coefficient $C_{X}$ and the optimum sail angle of attack $\alpha_{\text {opt }}$. With the current wind direction and course of the USV, the optimum turning angle $\psi$ can be calculated accordingly. Based on the reached optimum sail angle of attack, the sail automatic control system would adjust the angle of attack in a reasonable manner. Consequently, to reveal the relationship between $C_{X}$ and $\psi$ and then reach $\alpha_{\text {opt }}$ and $\psi$, Figure 5 manifests the aerodynamic characteristic of the sail in form of curve. In Figure 5, suppose the course of the USV passes through the origin of coordinate, and the direction of the relative wind speed $V_{A}$ coincides with the abscissa of the curve. When the value of the relative wind angle $\beta$ is fixed, $C_{X}$ can be reached by projecting one certain point $A$ into the course of the USV.

$$
\begin{aligned}
C_{X} & =C_{L} \sin \beta-C_{D} \cos \beta \\
& =f(\alpha, \lambda, \nu) \sin \beta-g(\alpha, \lambda, \nu) \cos \beta .
\end{aligned}
$$

Thus, the point $A$ can be treated as $\alpha_{\text {opt }}$ corresponding to $\beta$. The optimum sail angle of attack $\alpha_{\text {opt }}$ can be reached as follows:

$$
\frac{\partial f}{\partial \alpha} \sin \beta-\frac{\partial g}{\partial \alpha} \cos \beta=0 .
$$

Consequently, as shown in Figure 5, $180-(\beta+\psi)=90-\alpha$; then the optimum turning angle can be

$$
\psi_{\mathrm{opt}}=90-\beta+\alpha_{\mathrm{opt}} .
$$

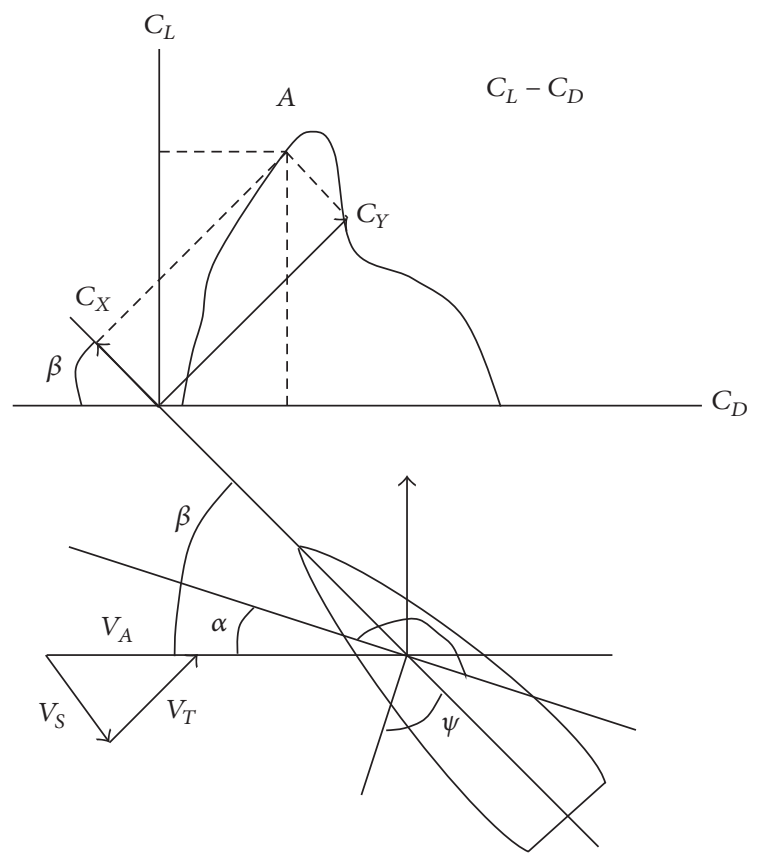

Figure 5: Aerodynamic characteristic of the sail in form of curve.

2.4.3. The Sail Angle of Attack Automatic Adjustment Algorithm. To design high-quality sail automatic control system, we propose one novel algorithm named the sail angle of attack automatic adjustment (Sail_4A). Within the Sail_4A algorithm, the raw data collected by the sensor system are processed via the bubble method $[30,31]$, and then the reliable data are chosen to represent the angle of the actual wind direction; then the optimum sail angle of attack is reached by using the procedure stated in Section 2.4.2; following that, compared with the current sail angle of attack, the sail automatic control system would adjust the sail with the amount of the corresponding optimum sail turning angle. Generally, sail-assisted facility can be used under certain conditional environment. In other words, the wind scale should live up to one certain range; then sail can be in effect accordingly. If the wind scale is small, then the sail-assisted USV cannot fulfill its energy-saving function. In the same way, if the wind scale exceeds the endurance of the sailassisted USV, then to ensure the navigation safety it is better to suspend the sail-assisted function in time. Consequently, to consolidate the robustness of our algorithm, we have limited the ranges of some parameters correspondingly. Suppose the threshold of wind speed ranges from $V_{\text {-wind_min }}$ to $V_{\text {-wind_max }}$; if the collected wind speed $V$ is out of the above range, then the Sail_4A algorithm is terminated automatically.

The pseudocode of the Sail_4A algorithm is summarized in Algorithm 1.

Algorithm 1 (Sail_4A).

Input:

$\theta_{\text {sail }}$-the current absolute angle of sail.

$\mathrm{C}_{\mathrm{USV}}$ - the heading angle of the USV. 
$\alpha$-the sail angle of attack.

Dir $_{\text {wind }}$ - the current wind direction.

\section{Parameters:}

$N$ - the number of wind direction data.

$V_{\text {_wind }}$-collected wind speed.

Data $_{\text {set }}$ - collection of 20 sets of data.

data $_{i}$ - the $i$ th wind direction data, $i=1,2,3,4$.

$\operatorname{avg}_{\text {val }}$ - the average value of four sets of data.

$\beta$-the relative wind direction angle between $V_{A}$ and $V_{S}$.

$\alpha_{\text {opt }}$ - the optimal sail angle of attack.

$\psi_{\text {opt }}$ - the optimal turning angle.

$\Delta_{\text {angle }}$ - the allowed turning angle error.

(1) DataDeal $\left(\theta_{\text {sail }}, C_{\mathrm{USV}}, \alpha, \operatorname{Dir}_{\text {wind }}\right)$

(2) $/{ }^{*}$ Perform the following operations for each DataDeal ${ }^{*} /$

(3) $/{ }^{*}$ Initialize data ${ }_{i}$ by performing Sort ()$^{*} /$

(4) $N=N+1$

(5) if $N==20$

(6) Sort $\left(\right.$ Data $\left._{\text {set }}\right)$

(7) if $V_{\text {-wind }}<V_{\text {_wind_min }}$ or $V_{\text {_wind }}>V_{\text {_wind_max }}$

(8) then return; \% Terminate the Sail_4A algo-

rithm

(9) end

(10) $/{ }^{*}$ Calculate the optimal angle of attack ${ }^{*} /$

(11) $\quad \operatorname{avg}_{\text {val }}=\operatorname{sum}\left(\right.$ data $\left._{i}\right) / 4$

(12) $\alpha_{\text {opt }}$ calculated by equation (5).

(13) $\beta=\operatorname{avg}_{\text {val }}-\alpha_{\text {opt }}$

(14) $\psi_{\mathrm{opt}}=90-\beta+\alpha_{\mathrm{opt}}$

(15) $/{ }^{*}$ Adjust the angle of attack * $/$

(16) while $\alpha-\alpha_{\text {opt }}>\Delta_{\text {angle }}$ do

(17) $\alpha=\alpha-\Delta_{\text {angle }}$

(18) end while

(19) while $\alpha-\alpha_{\text {opt }}<-\Delta_{\text {angle }}$ do

(20) $\alpha=\alpha+\Delta_{\text {angle }}$

(21) end while

(22) end if

As shown in the Sail_4A algorithm, if the wind scale is not fit for the sail-assisted application, then the algorithm is suspended. The data size $N$ can be adjusted according to the requirements of the SUICS. Referring to the number of wind direction data $N$, after several times of sail-assisted USV trials, we find that $N=20$ is enough for the experiments.

Generally, the ranges of the parameters related to the sailassisted algorithm Sail_4A are determined by wind tunnel test. According to wind tunnel test reported in [4], the usable range of the sail attack angle $\alpha$ is between $-5^{\circ}$ and $90^{\circ} . \theta_{\text {sail }}$, $C_{\text {USV }}, \operatorname{Dir}_{\text {wind }}, V_{\text {-wind }}$, and $\alpha$ are measured by our sensor systems. After a certain number of data collections, Data ${ }_{\text {set }}$ is reached. Consequently, data ${ }_{i}$, avg $_{\text {val }}$, and $\beta$ can be obtained by way of computing. $\alpha_{\text {opt }}$ and $\psi_{\text {opt }}$ are reached by the embedded functions of several commercial software programs including Matlab and Lingo.

By using the collected data, with the aid of our algorithm, when the optimum sail angle of attack $\alpha_{\text {opt }}$ is reached, the optimal turn angle can be realized within the acceptable error $\Delta_{\text {angle }}$. Referring to the complexity of our algorithm, the increased calculation time mainly generated from three parts. The first part is generated from the time used for processing the collected data based on the bubble method with complexity of $N \times N$ ( $N$ is the data size). The second part is the time to compute the optimum sail angle of attack $\alpha_{\text {opt }}$. The third part is the time to adjust the sail angle of attack. As the optimum sail angle of attack $\alpha_{\text {opt }}$ can be obtained through certain embedded functions in several commercial software programs, such as Matlab and Lingo, then the value of $\alpha_{\text {opt }}$ can be achieved by using their embedded functions instantaneously. When it comes to the adjustment time, it is related to the mechanical behavior of the equipment; it takes little time to realize the optimum sail angle of attack in essence. Consequently, it can be obtained that the computational time complexity is equal to $O(N \times N)$.

\section{The Implementation Process of the SUICS}

3.1. Implementation of the Communication System. Generous researchers have stressed the work related to the machine vision by using the microprocessors. Reference [32] implemented a low cost matching motion estimation sensor based on the Nios II microprocessor. By using a custom instructionbased paradigm on a Nios II microprocessor, [33] successfully accelerated the block-matching algorithms. Many sensors have been applied to the industrial fields, [34] fulfilled automated guided vehicle system based on laser guidance, and automatic parking system was introduced in [35] with the aid of bioinspired $1 \mathrm{D}$ optical flow sensors.

Referring to the SUICS, our communication system adopts the CHD-T5 Wi-Fi video module which is compatible with IEEEE802.11b/g/n standard. Compared with other similar Wi-Fi modules, including the advanced LSD4WF2MD05107 Wi-Fi module, CHD-T5 Wi-Fi has lots of advantages over other similar Wi-Fi modules. Firstly, our module supports generous functions, including $\mathrm{Wi}-\mathrm{Fi}$ to video, $\mathrm{Wi}$ $\mathrm{Fi}$ to serial port, Wi-Fi to GPIO, and Wi-Fi to I2C. Free drive USB camera can access our module and collect wireless video data. Following that, taking into account that $\mathrm{CHD}$ T5 is compatible with lots of models including AP, STA, and AP \& STA, CHD-T5 satisfies with the requirements of a variety of application environments. Based on MIPS24KE, our communication system has the clock speed up to $360 \mathrm{MHz}$ (the wireless rate of the $\mathrm{Wi}-\mathrm{Fi}$ is $150 \mathrm{Mbps}$ ) and $256 \mathrm{Mbit}$ SDRAM. Finally, compared to some other Wi-Fi modules on the market including the popular LSD4WF-2MD05107 Wi-Fi module, the CHD-T5 is smaller and more powerful and has a 


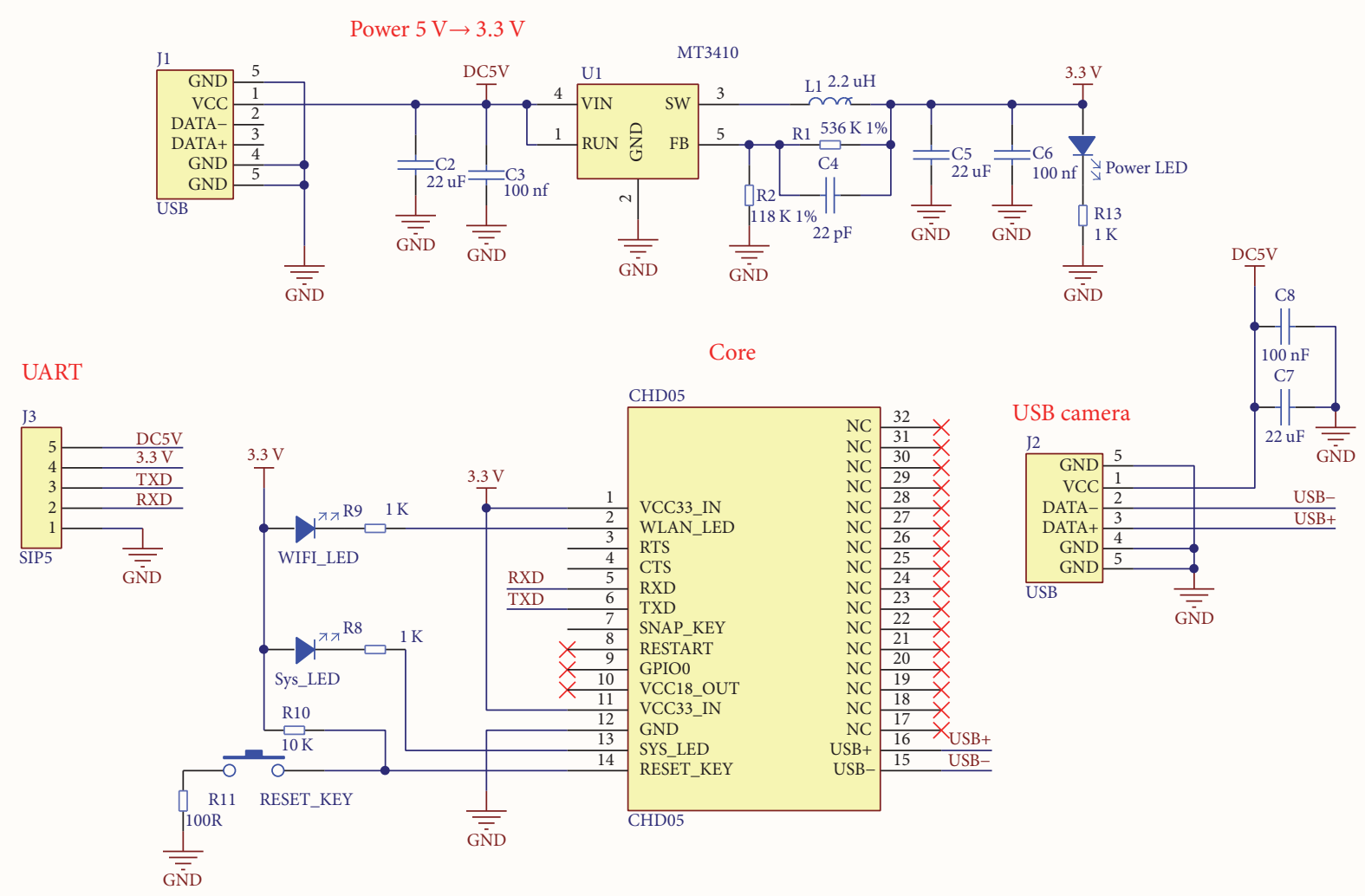

FIgURE 6: Schematic diagram of bottom plate of the communication system.

larger data throughput. It is widely used in image analysis, pipeline endoscopy, intelligent robots, and other fields. By virtue of the excellent hardware performance, our communication system can achieve video image transmission with various formats and resolution, support the free-drive camera with the USB UVC standard, and provide various video performance parameters adjustment interfaces.

Figure 6 show the schematic diagram of bottom plate of the communication system. Within Figure 6, SYS_LED is the system indicator, it would flash when it is controlled, and it will flash for $3 \mathrm{~s}$ when under the boot module. RUN_LED is often bright under AP mode; it is often bright when connected to router under STA mode successfully. RFS_KEY restores the factory settings when it is pressed for $5 \mathrm{~s}$. SNAP_KEY is the camera button, after the trigger photos are sent to all the connections to the client. TXD is the serial port to send; RXD is the serial port to accept.

\subsection{Implementation of the Senor System}

3.2.1. Implementation of the Wind Sensor System. The wind sensors mounted on our sail-assisted USV belong to the hand-held devices. One default is that the wind direction and speed cannot be transferred in the form of electrical signals receivable by other controllers. Therefore, we have

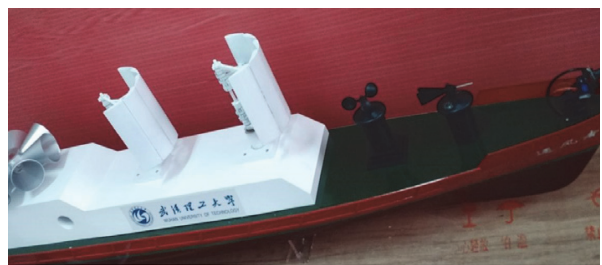

FIGURE 7: The wind sensor system of the SUICS.

redesigned the wind direction and speed sensors based on the mechanical structure of the instruments. When it comes to the wind direction sensor, as the wind vane senses the wind direction, the rotary potentiometer drives and generates the linear resistance value and obtains corresponding wind direction by using the $\mathrm{AD}$ conversion. The wind speed is reached by above similar approach. Figure 7 shows the redesigned wind sensor system installed on the stem of our sail-assisted USV.

3.2.2. Implementation of the Ultrasonic Sensor System. The ultrasonic module adopts the HC-SR04 module. Its product parameters are presented as follows. Its typical operating voltage is $5 \mathrm{~V}$, ultrasmall static operating current is less than 


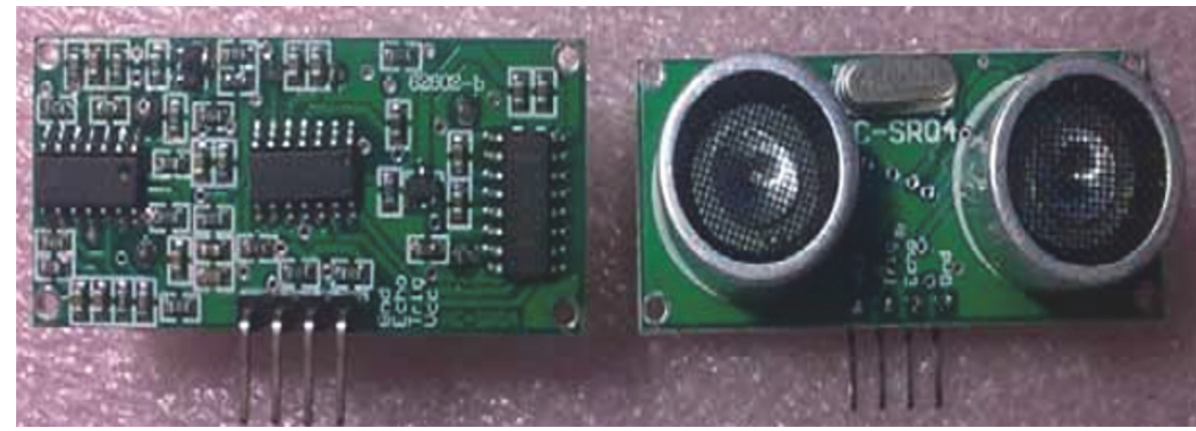

FIGURE 8: Ultrasonic distance measuring module.

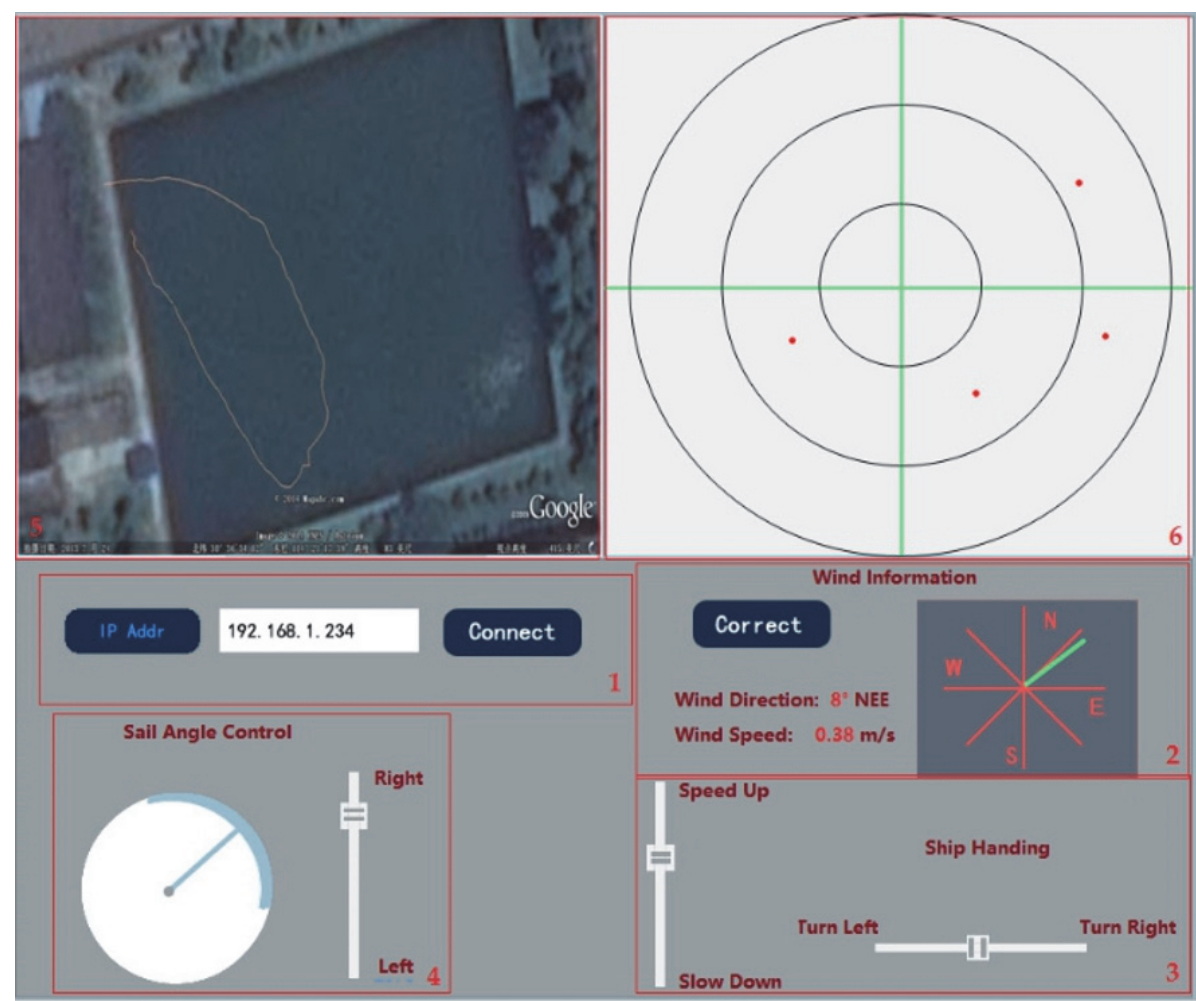

FIGURE 9: PC software screenshot.

$2 \mathrm{~mA}$, induction angle is no more than $15^{\circ}$, detection range is from $2 \mathrm{~cm}$ to $400 \mathrm{~cm}$, and high precision can be up to $0.3 \mathrm{~cm}$ and is completely compatible with the $\mathrm{GH}-311$ security module. Figure 8 shows the developed ultrasonic sensor system in our SUICS.

Working principle of the ultrasonic sensor system is illustrated as follows. At first, IO is used to trigger range, while the high level signal at least lasts for $10 \mu \mathrm{s}$. Then, the system automatically sends $40 \mathrm{KHz}$ square wave 8 times and detects whether there is a signal returned. Once the signal returned, the duration time of the high level signal can be recorded as the time of the ultrasonic wave from launch to return. Consequently, the range can be $1 / 2$ of the above time multiplied by the acoustic velocity.
3.3. Implementation of the PC Platform. According to the design introduced in Section 2.3, the PC platform has been implemented as shown in Figure 9. Our PC software interface is divided into 6 functional modules. Module 1 is the communication connection module, which is used to connect the host computer and the communication system. Module 2 is the wind speed and direction information display window, the "Correct" button is used to initialize and correct the wind sensors. Module 3 is an USV control module; the module includes two drag bars, which is used to control the speed and direction of the USV. Module 4 is the sail angle control module, which is used to view and manually control the angle of attack. Module 5 is the track display module, which is used to track the trajectory of the track. Module 6 is 


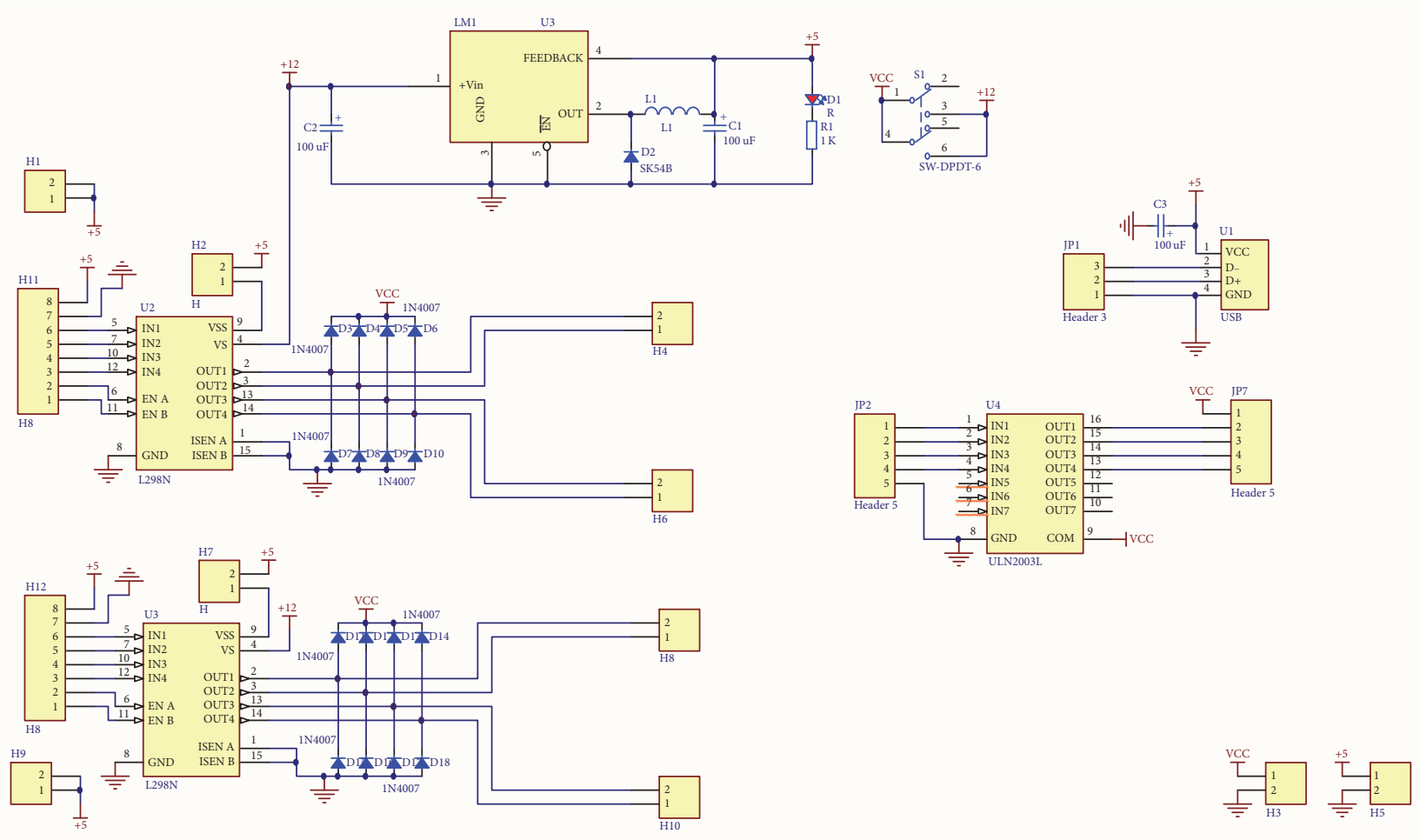

Figure 10: Principle diagram of drive module.

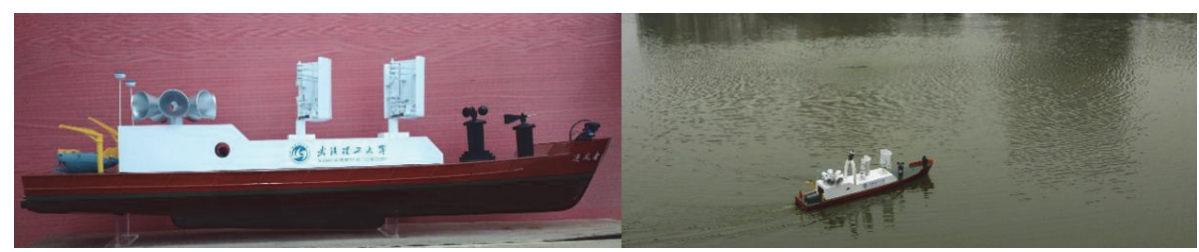

FIGURE 11: Overall figure of the test boat.

an obstacle display module, which can display the obstacles around the vehicle.

\subsection{Implementation of the Drive System}

3.4.1. Implementation of the Servo Module. When it comes to the servo, the control signal is entered into the signal modulation chip by the channel of the receiver, and the DC bias voltage is obtained. It has an internal reference circuit that produces a reference signal with a period of $20 \mathrm{~ms}$ and width of $1.5 \mathrm{~m}$. Then, comparing the voltage of the DC bias with the voltage of the potentiometer, the output voltage difference can be calculated. Finally, the difference between the positive and negative output voltage is 0 ; then the motor would stop operating.

3.4.2. Implementation of the Servo Drive Module. The principle diagram of the servo drive module is shown in Figure 10. It can be seen that the servo drive module integrates two chips with the type of L298N and could drive four DC motors or two stepper motors. Meanwhile, the servo drive module also integrates one ULN2003 that can drive a stepper motor or four DC motors. Also, the DC-DC LM2596 chip, the steering engine interface, and the USB socket are integrated in the servo drive module.

\section{Experiments of the Sail-Assisted USV with the SUICS}

4.1. Experiment Scheme. As shown in Figure 11, by using our laboratory USV, the SUICS has been designed and implemented according to the details illustrated in Sections 2 and 3.

To validate the performance of the SUICS, the water tests should be carried out reasonably under the sound experiment scheme. In addition to the SUICS, our sail-assisted USV has been equipped with the GPS and other auxiliary sets to ensure that the experiments are conducted smoothly. The experiment scheme is listed as follows. 
TABLE 2: Wind speed and direction that the sail-assisted USV experienced.

\begin{tabular}{|c|c|c|c|}
\hline Time & Wind direction $\left(^{\circ}\right)$ & Wind speed $(\mathrm{m} / \mathrm{s})$ & Wave height $(\mathrm{m})$ \\
\hline $15: 56: 31$ & 292.5 & 0.1 & 0 \\
\hline $15: 56: 33$ & 292.5 & 0.2 & 0 \\
\hline $15: 56: 37$ & 337.5 & 0.0 & 0 \\
\hline $15: 56: 39$ & 337.5 & 0.0 & 0 \\
\hline $15: 56: 42$ & 337.5 & 0.0 & 0 \\
\hline $15: 56: 49$ & 315 & 0.1 & 0 \\
\hline $15: 56: 53$ & 315 & 0.2 & 0 \\
\hline $15: 56: 55$ & 337.5 & 0.8 & 0.1 \\
\hline $15: 56: 59$ & 0 & 0.18 & 0 \\
\hline $15: 57: 01$ & 0 & 0.26 & 0.1 \\
\hline $15: 57: 04$ & 0 & 0.29 & 0.1 \\
\hline $15: 57: 08$ & 337.5 & 0.32 & 0.1 \\
\hline $15: 57: 11$ & 337.5 & 0.33 & 0.1 \\
\hline $15: 57: 14$ & 0 & 0.29 & 0.1 \\
\hline $15: 57: 18$ & 45 & 0.35 & 0.1 \\
\hline $15: 57: 20$ & 67.5 & 0.36 & 0.1 \\
\hline $15: 57: 23$ & 45 & 0.30 & 0.1 \\
\hline $15: 57: 27$ & 45 & 0.30 & 0.1 \\
\hline $15: 57: 33$ & 67.5 & 0.0 & 0 \\
\hline $15: 57: 33$ & 67.5 & 0.19 & 0 \\
\hline $15: 57: 36$ & 67.5 & 0.18 & 0 \\
\hline $15: 57: 40$ & 90 & 0.23 & 0.1 \\
\hline $15: 57: 42$ & 90 & 0.30 & 0.1 \\
\hline $15: 57: 46$ & 112.5 & 0.28 & 0.1 \\
\hline $15: 57: 49$ & 135 & 0.15 & 0 \\
\hline $15: 57: 52$ & 270 & 0.5 & 0.1 \\
\hline $15: 57: 55$ & 292.5 & 0.15 & 0 \\
\hline $15: 57: 58$ & 315 & 0.11 & 0 \\
\hline 15:58:01 & 292.5 & 0.0 & 0 \\
\hline $15: 58: 04$ & 315 & 0.47 & 0.1 \\
\hline $15: 58: 08$ & 0 & 0.38 & 0.1 \\
\hline 15:58:11 & 45 & 0.45 & 0.1 \\
\hline $15: 58: 14$ & 67.5 & 0.28 & 0.1 \\
\hline $15: 58: 17$ & 22.5 & 0.0 & 0 \\
\hline $15: 58: 20$ & 22.5 & 0.7 & 0.1 \\
\hline $15: 58: 24$ & 0 & 0.14 & 0 \\
\hline $15: 58: 26$ & 337.5 & 0.16 & 0 \\
\hline $15: 58: 29$ & 315 & 0.18 & 0 \\
\hline $15: 58: 32$ & 337.5 & 0.0 & 0 \\
\hline $15: 58: 36$ & 337.5 & 0.5 & 0.1 \\
\hline
\end{tabular}

Firstly, we install the SUICS on our sail-assisted USV and operate the sail in the lake or tank automatically and manually under wind condition. Following that, the USV could move forward and turn left and right with the aid of the sail. During navigation, by using the SUICS, the wind data are recorded and the optimum sail angle of attack can be calculated under different scenarios. Finally, the sustainable wind energy can be efficiently used by steering to the optimum sail angle of attack.
TABLE 3: Trajectory coordinates of the sail-assisted USV.

\begin{tabular}{lcc}
\hline Time & Latitude & Longitude \\
\hline $15: 56: 31$ & $30^{\circ} 36^{\prime} 47.12^{\prime \prime} \mathrm{N}$ & $114^{\circ} 21^{\prime} 05.05^{\prime \prime} \mathrm{E}$ \\
$15: 56: 37$ & $30^{\circ} 36^{\prime} 46.91^{\prime \prime} \mathrm{N}$ & $114^{\circ} 21^{\prime} 05.15^{\prime \prime} \mathrm{E}$ \\
$15: 56: 43$ & $30^{\circ} 36^{\prime} 46.69^{\prime \prime} \mathrm{N}$ & $114^{\circ} 21^{\prime} 05.26^{\prime \prime} \mathrm{E}$ \\
$15: 56: 49$ & $30^{\circ} 36^{\prime} 46.84^{\prime \prime} \mathrm{N}$ & $114^{\circ} 21^{\prime} 05.62^{\prime \prime} \mathrm{E}$ \\
$15: 56: 55$ & $30^{\circ} 36^{\prime} 46.95^{\prime \prime} \mathrm{N}$ & $114^{\circ} 21^{\prime} 05.68^{\prime \prime} \mathrm{E}$ \\
$15: 57: 01$ & $30^{\circ} 36^{\prime} 47.14^{\prime \prime} \mathrm{N}$ & $114^{\circ} 21^{\prime} 05.61^{\prime \prime} \mathrm{E}$ \\
$15: 57: 07$ & $30^{\circ} 36^{\prime} 47.25^{\prime \prime} \mathrm{N}$ & $114^{\circ} 21^{\prime} 05.37^{\prime \prime} \mathrm{E}$ \\
$15: 57: 13$ & $30^{\circ} 36^{\prime} 47.19^{\prime \prime} \mathrm{N}$ & $114^{\circ} 21^{\prime} 05.23^{\prime \prime} \mathrm{E}$ \\
$15: 57: 19$ & $30^{\circ} 36^{\prime} 47.04^{\prime \prime} \mathrm{N}$ & $114^{\circ} 21^{\prime} 05.25^{\prime \prime} \mathrm{E}$ \\
$15: 57: 25$ & $30^{\circ} 36^{\prime} 47.00^{\prime \prime} \mathrm{N}$ & $114^{\circ} 21^{\prime} 05.38^{\prime \prime} \mathrm{E}$ \\
$15: 57: 31$ & $30^{\circ} 36^{\prime} 47.10^{\prime \prime} \mathrm{N}$ & $114^{\circ} 21^{\prime} 05.39^{\prime \prime} \mathrm{E}$ \\
$15: 57: 37$ & $30^{\circ} 36^{\prime} 47.20^{\prime \prime} \mathrm{N}$ & $114^{\circ} 21^{\prime} 05.52^{\prime \prime} \mathrm{E}$ \\
$15: 57: 43$ & $30^{\circ} 36^{\prime} 47.11^{\prime \prime} \mathrm{N}$ & $114^{\circ} 21^{\prime} 05.59^{\prime \prime} \mathrm{E}$ \\
$15: 57: 49$ & $30^{\circ} 36^{\prime} 47.03^{\prime \prime} \mathrm{N}$ & $114^{\circ} 21^{\prime} 05.57^{\prime \prime} \mathrm{E}$ \\
$15: 57: 55$ & $30^{\circ} 36^{\prime} 46.97^{\prime \prime} \mathrm{N}$ & $114^{\circ} 21^{\prime} 05.48^{\prime \prime} \mathrm{E}$ \\
$15: 58: 01$ & $30^{\circ} 36^{\prime} 46.95^{\prime \prime} \mathrm{N}$ & $114^{\circ} 21^{\prime} 05.58^{\prime \prime} \mathrm{E}$ \\
$15: 58: 07$ & $30^{\circ} 36^{\prime} 47.09^{\prime \prime} \mathrm{N}$ & $114^{\circ} 21^{\prime} 05.62^{\prime \prime} \mathrm{E}$ \\
$15: 58: 13$ & $30^{\circ} 36^{\prime} 47.20^{\prime \prime} \mathrm{N}$ & $114^{\circ} 21^{\prime} 05.32^{\prime \prime} \mathrm{E}$ \\
$15: 58: 19$ & $30^{\circ} 36^{\prime} 47.22^{\prime \prime} \mathrm{N}$ & $114^{\circ} 21^{\prime} 05.17^{\prime \prime} \mathrm{E}$ \\
$15: 58: 25$ & $30^{\circ} 36^{\prime} 47.08^{\prime \prime} \mathrm{N}$ & $114^{\circ} 21^{\prime} 05.01^{\prime \prime} \mathrm{E}$ \\
\hline
\end{tabular}

4.2. Experiment Results and Discussion. Under different wind speeds and directions, the experiments are carried out on the Shuiyun lake of Wuhan University of Technology shown in Figure 12 to verify the performance of the SUICS. During the experimental period, the related wind data that the sailassisted USV experienced are recorded in Table 2, its trajectory coordinates are given in Table 3 , and the corresponding trajectories are depicted in Figure 12.

As shown in Table 2, the wind speed was about $0.2 \mathrm{~m} / \mathrm{s}$, the initial heading of the sail-assisted USV was $283^{\circ}$, and the relative wind direction was $292^{\circ}$. The average velocity of the sail-assisted USV was $0.3 \mathrm{~m} / \mathrm{s}$. The USV was sailing before the wind at about 15:57:20, by using the Sail_4A algorithm embedded in the SUICS, the sail was adjusted automatically, and then the velocity of the sail-assisted USV reached its maximum value. When sliding until 15:57:50, the USV was sailing against the wind, owing to the limited thrust generated by the sail; its velocity decreased a little. Following that, the sail automatic control system of the SUICS adjusted the sail of attack at 15:58:08 with the Sail_4A algorithm; the velocity of the USV began to rise once again. The entire process of the sailing angle adjustment lasted $3 \mathrm{~s}$, and the USV travelled almost $1 \mathrm{~m}$.

It can be seen from Figure 12 that our sail-assisted USV navigates from the first point labeled as "1" to the second point labeled as " 2 " and finally to the 20 th point labeled as "20." With lots of steering control, straight line navigation, and Zigzag routing, the maneuverability of the sail-assisted USV is validated through the above actions remarkably. Consequently, the effectiveness of our SUICS is examined and verified successfully. 

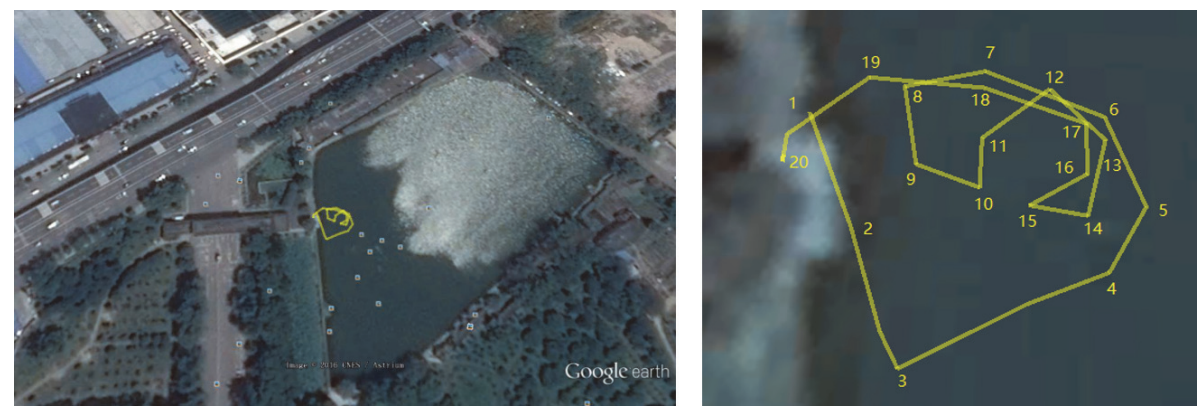

FIGURE 12: Navigation trajectory of the sail-assisted USV.

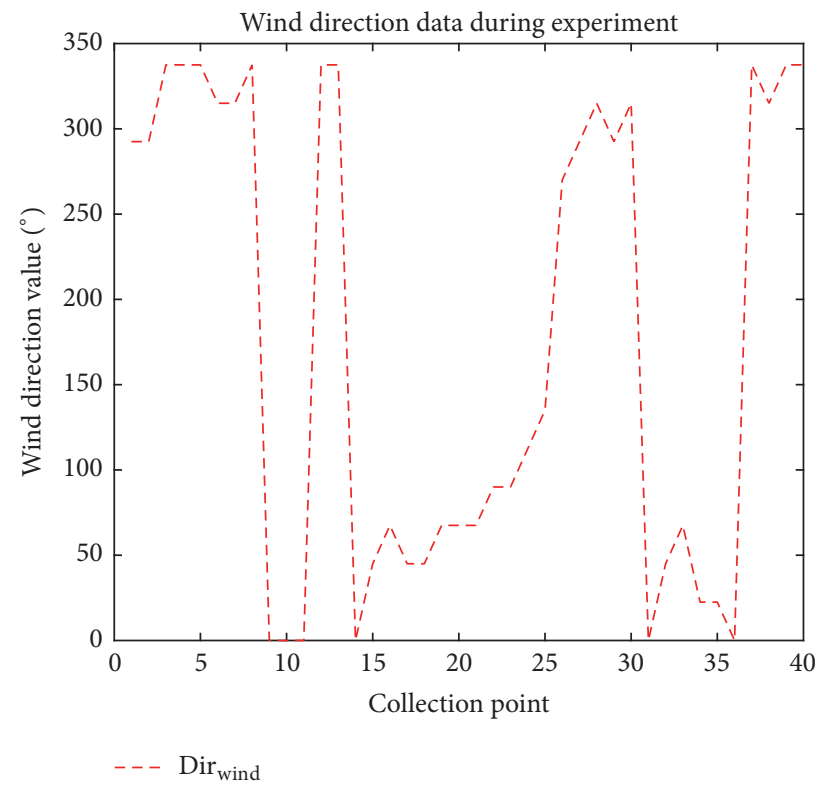

FIGURE 13: Wind direction $\mathrm{Dir}_{\text {wind }}$ versus sampling point.

The wind direction $\operatorname{Dir}_{\text {wind }}$ versus sampling point, the wind speed $V_{T}$ versus sampling point, and the wave height versus sampling point are presented in our manuscript. Figures 13, 14, and 15 correspond to the above illustration in sequence.

During navigation, wind direction $\mathrm{Dir}_{\text {wind }}$ is collected by sensor system of the sail-assisted USV. Figure 13 shows that $\mathrm{Dir}_{\text {wind }}$ is varied with sampling point and falls in $\left[0360^{\circ}\right]$, where $0^{\circ}$ and $360^{\circ}$ are overlapped. Figure 14 gives wind speed $V_{T}$ versus sampling point. It can be seen that $V_{T}$ ranges from 0 to $0.8 \mathrm{~m} / \mathrm{s}$ and with mean value 0.24 and variance value 0.03 . Consequently, as depicted in Figure 14 sampled wind speed $V_{T}$ is stable. Wave height $H_{\text {wave }}$ versus sampling point is demonstrated in Figure 15. Within our SUICS, the precision of wave height is $0.1 \mathrm{~m}$; thus the numbers of $H_{\text {wave }}$ in Figure 15 are 0 and 0.1 .

The experiments demonstrate that the PC platform is human-machine interface friendly, the sensor system is with high data collection efficiency, the information can be transferred simultaneously without error, and the lower machine platform can respond to the corresponding instructions

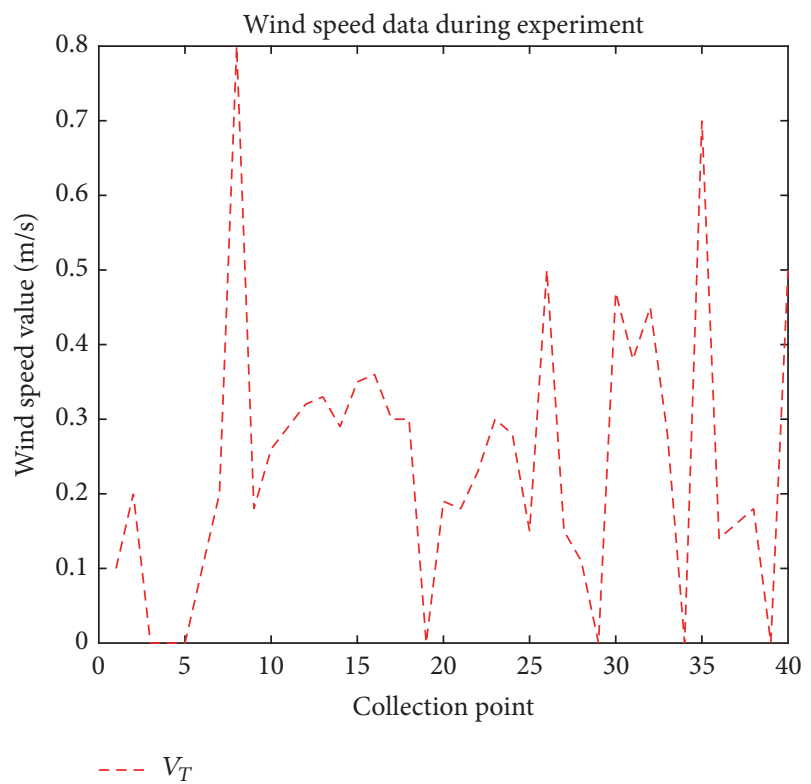

FIgUre 14: Wind speed $V_{T}$ versus sampling point.

fleetly. Benefiting from our proposed Sail_4A algorithm, the sail automatic control system adjusts the sail angle of attack automatically with the aid of the sensor system, the PC platform, the communication system, and the drive system of the SUICS. Those experiments validate that our SUICS is reliable, efficient, and intelligent.

\section{Conclusions}

The sail-assisted USV intelligent control system has been designed and implemented. With our SUICS, the wind speed and direction and the environment information of the USV are recorded in real time; the information transferred reliably and smoothly among the subsystems; the PC platform owns the friendly human-machine interface; the lower machine platform can respond to the instructions timely and effectively, and then the optimal sail angle of attack is achieved based on our proposed Sail_4A algorithm. The sustainable wind energy is exploited fully by using the designed SUICS. Experiments under wind condition verify the excellent performance of the SUICS. In the near future, 


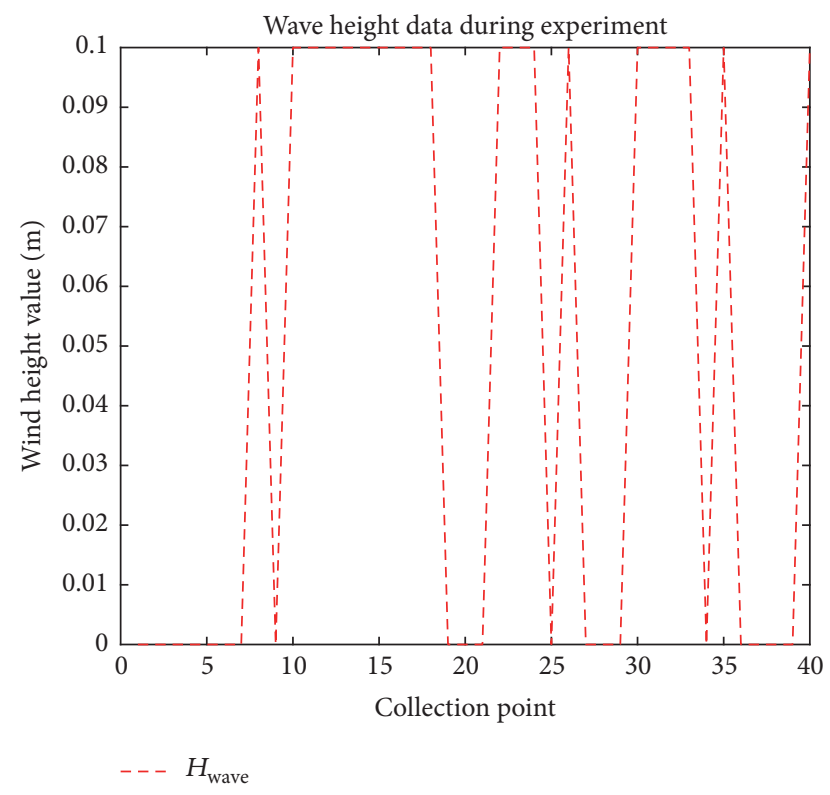

FIGURE 15: Wave height $H_{\text {wave }}$ versus sampling point.

we will apply our SUICS to the merchant ship to explore the ocean sustainable wind energy; then the energy consumption of the shipping industry would lower down substantially to cater for the requirements issued by IMO.

\section{Competing Interests}

The authors declare that there is no conflict of interests regarding the publication of this paper.

\section{Authors' Contributions}

Yong Ma conceived and designed the SUICS framework; Yong Ma and Yujiao Zhao wrote the paper; Yujiao Zhao, Jiantao Diao, Langxiong Gan, Huaxiong $\mathrm{Bi}$, and Jingming Zhao performed the experiments and analyzed the data.

\section{Acknowledgments}

The authors are partially supported by the National Science Foundation of China (51309186, 61304043, 51479156, 51579202, 71501151, and 61673223), China Postdoctoral Science Foundation Funded Project (2014M560633, 2015M571788, and 2015T80848), the Fund of Hubei Inland Shipping Technology Key Laboratory (NHHY2014003), and the General Program of the Provincial Natural Science Foundation (2014CFB856).

\section{References}

[1] R. Saidur, N. A. Rahim, M. R. Islam, and K. H. Solangi, "Environmental impact of wind energy," Renewable and Sustainable Energy Reviews, vol. 15, no. 5, pp. 2423-2430, 2011.

[2] J. Heng, C. Wang, X. Zhao, and L. Xiao, "Research and application based on adaptive boosting strategy and modified
CGFPA algorithm: a case study for wind speed forecasting," Sustainability, vol. 8, no. 3, pp. 235-259, 2016.

[3] F. Y. Zhang, Y. Q. Dong, and K. Q. Zhang, "A novel combined model based on an artificial intelligence algorithm-a case study on wind speed forecasting in Penglai, China," Sustain, vol. 8, pp. 555-574, 2016.

[4] J. H. He, Y. H. Hu, J. J. Tang, and S. Y. Xue, "Research on sail aerodynamics performance and sail-assisted ship stability," Journal of Wind Engineering and Industrial Aerodynamics, vol. 146, pp. 81-89, 2015.

[5] Q. Li, Y. Nihei, T. Nakashima, and Y. Ikeda, "A study on the performance of cascade hard sails and sail-equipped vessels," Ocean Engineering, vol. 98, pp. 23-31, 2015.

[6] Y. Ma, H. Wang, L. Gan, M. Guo, L. Huang, and J. Zhang, "Mobile robots path planning using the overall conflict resolution and time baseline coordination," Mathematical Problems in Engineering, vol. 2014, Article ID 902587, 13 pages, 2014.

[7] Y. Ma, M. Zamirian, Y. D. Yang, Y. Xu, and J. Zhang, "Path planning for mobile objects in four-dimension based on particle swarm optimization method with penalty function," Mathematical Problems in Engineering, vol. 2013, Article ID 613964, 9 pages, 2013.

[8] Y. Ma, H. Wang, Y. Xie, and M. Guo, "Path planning for multiple mobile robots under double-warehouse," Information Sciences, vol. 278, pp. 357-379, 2014.

[9] L. A. Danao, J. Edwards, O. Eboibi, and R. Howell, "A numerical investigation into the influence of unsteady wind on the performance and aerodynamics of a vertical axis wind turbine," Applied Energy, vol. 116, pp. 111-124, 2014.

[10] S. Roy and U. K. Saha, "Wind tunnel experiments of a newly developed two-bladed Savonius-style wind turbine," Applied Energy, vol. 137, pp. 117-125, 2015.

[11] V. G. Chapin, N. De Carlan, and P. Heppel, "Performance optimization of interacting sails through fluid structure coupling," in Proceedings of the 2nd International Conference on Innovation in High Performance Sailing Yachts, pp. 75-87, Lorient, France, July 2010.

[12] H. Renzsh and K. Graf, "Fluid-structure interaction simulation of spinnakers getting closer to reality," in Proceedings of the 2nd International Conference on Innovation in High Performance Sailing Yachts, Lorient, France, 2010.

[13] F. C. Gerhardt, R. G. J. Flay, and P. Richards, "Unsteady aerodynamics of two interacting yacht sails in two-dimensional potential flow," Journal of Fluid Mechanics, vol. 668, pp. 551-581, 2011.

[14] R. Leloup, K. Roncin, G. Bles, J.-B. Leroux, C. Jochum, and Y. Parlier, "Kite and classical rig sailing performance comparison on a one design keel boat," Ocean Engineering, vol. 90, pp. 3948, 2014.

[15] A. Balasubramanian, R. Mahajan, A. Venkataramani, B. N. Levine, and J. Zahorjan, "Interactive wifi connectivity for moving vehicles," ACM SIGCOMM Computer Communication Review, vol. 38, no. 4, pp. 427-438, 2008.

[16] O. Sarbishei and K. Radecka, "Analysis of Mean-Square-Error (MSE) for fixed-point FFT units," in Proceedings of the IEEE International Symposium of Circuits and Systems (ISCAS '11), pp. 1732-1735, IEEE, Rio de Janeiro, Brazil, May 2011.

[17] A. Valdessalici, G. Frassi, and A. Bellini, "Efficient implementation of a spectrum analyzer for fixed point architectures," in Proceedings of the IEEE International Conference on Acoustics, Speech, and Signal Processing (ICASSP '05), pp. V109-V112, March 2005. 
[18] G. Botella, U. Meyer-Baese, A. García, and M. Rodríguez, "Quantization analysis and enhancement of a VLSI gradientbased motion estimation architecture," Digital Signal Processing, vol. 22, no. 6, pp. 1174-1187, 2012.

[19] G. Botella, A. García, M. Rodríguez-Álvarez, E. Ros, U. MeyerBaese, and M. C. Molina, "Robust bioinspired architecture for optical-flow computation," IEEE Transactions on Very Large Scale Integration (VLSI) Systems, vol. 18, no. 4, pp. 616-629, 2010.

[20] A. Shojaeifard, F. Zarringhalam, and M. Shikh-Bahaei, "Joint physical layer and data link layer optimization of CDMA-based networks," IEEE Transactions on Wireless Communications, vol. 10, no. 10, pp. 3278-3287, 2011.

[21] M. Conti and S. Giordano, "Mobile ad hoc networking: milestones, challenges, and new research directions," IEEE Communications Magazine, vol. 52, no. 1, pp. 85-96, 2014.

[22] Y. Q. Zhu, B. Chen, M. Qin, and Q. A. Huang, "2-D micromachined thermal wind sensors-a review," IEEE Internet of Things Journal, vol. 1, pp. 216-232, 2014.

[23] A. A. Quarta and G. Mengali, "Trajectory approximation for low-performance electric sail with constant thrust angle," Journal of Guidance, Control, and Dynamics, vol. 36, no. 3, pp. 884-887, 2013.

[24] H. Zeng, "Discuss AT89C51 single-chip microcomputer in the application of SR motor control," Electron-Test, vol. 5, pp. 123124, 2013.

[25] C. B. Hübschle, G. M. Sheldrick, and B. Dittrich, "ShelXle: a Qt graphical user interface for SHELXL," Journal of Applied Crystallography, vol. 44, no. 6, pp. 1281-1284, 2011.

[26] M. Schoop, F. Köhne, and K. Ostertag, "Communication quality in business negotiations," Group Decision and Negotiation, vol. 19, no. 2, pp. 193-209, 2010.

[27] H. Ortega-Arranz, Y. Torres, A. Gonzalez-Escribano, and D. R. Llanos, "TuCCompi: a multi-layer model for distributed heterogeneous computing with tuning capabilities," International Journal of Parallel Programming, vol. 43, no. 5, pp. 939-960, 2015.

[28] Y. K. Cai, Z. Q. Liu, Z. Y. Shi, Q. H. Song, and Y. Wan, "Influence of machined surface roughness on thrust performance of micro-nozzle manufactured by micro-milling," Experimental Thermal and Fluid Science, vol. 77, pp. 295-305, 2016.

[29] P. Toivanen, P. Janhunen, and J. Envall, "Electric solar wind sail control and navigation," Advances in the Astronautical Sciences, vol. 145, pp. 275-285, 2012.

[30] T. Araki and Y. Kikuchi, "Hamiltonian laceability of bubble-sort graphs with edge faults," Information Sciences, vol. 177, no. 13, pp. 2679-2691, 2007.

[31] J. Harris and D. Barber, "Speech and gesture interfaces for squad-level human-robot teaming," in Proceedings of the Unmanned Systems Technology XVI, vol. 9084 of Proceedings of SPIE, International Society for Optics and Photonics, Baltimore, Md, USA, May 2014.

[32] D. González, G. Botella, U. Meyer-Baese et al., "A low cost matching motion estimation sensor based on the NIOS II microprocessor," Sensors, vol. 12, no. 10, pp. 13126-13149, 2012.

[33] D. González, G. Botella, C. García, M. Prieto, and F. Tirado, "Acceleration of block-matching algorithms using a custom instruction-based paradigm on a Nios II microprocessor," EURASIP Journal on Advances in Signal Processing, vol. 2013, no. 1, article 118, pp. 1-20, 2013.

[34] L. Schulze and A. Wüllner, "The approach of automated guided vehicle systems," in Proceedings of the IEEE International
Conference on Service Operations and Logistics, and Informatics (SOLI '06), pp. 522-527, IEEE, June 2006.

[35] S. Mafrica, A. Servel, and F. Ruffier, "Towards an automatic parking system using bio-inspired 1-D optical flow sensors," in Proceedings of the IEEE International Conference on Vehicular Electronics and Safety (ICVES '15), IEEE, Yokohama, Japan, November 2015. 


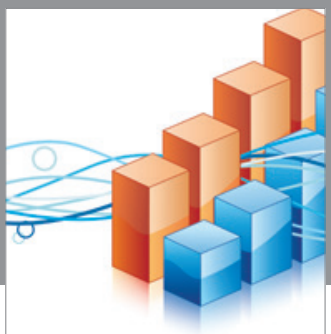

Advances in

Operations Research

vatem alat4

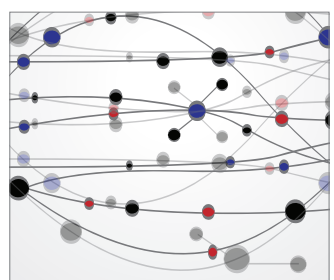

\section{The Scientific} World Journal
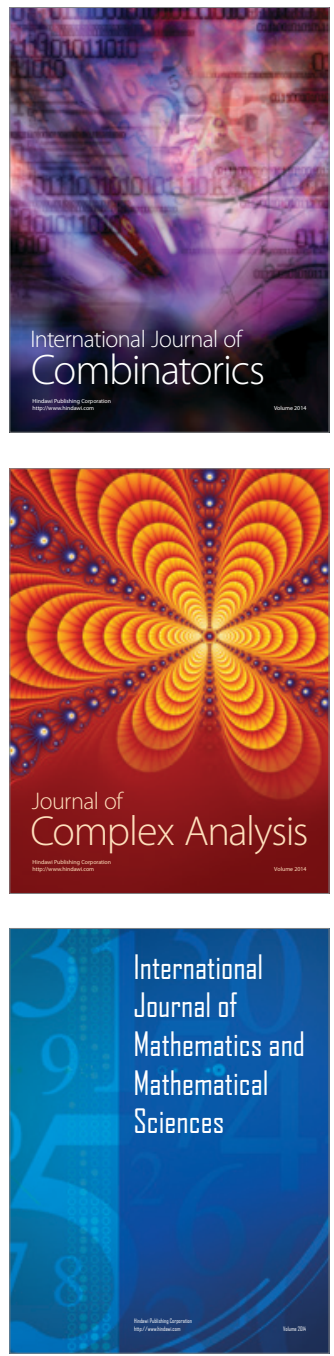
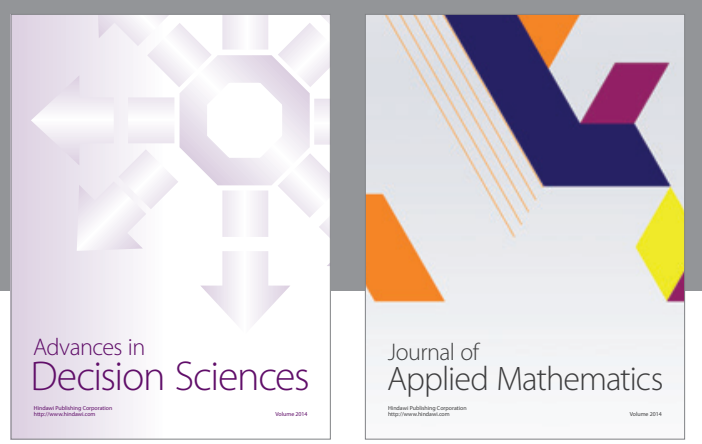

Algebra

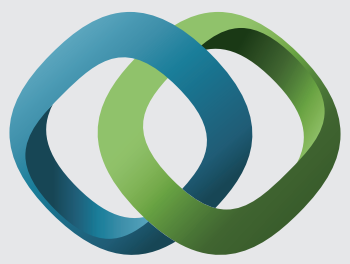

\section{Hindawi}

Submit your manuscripts at

http://www.hindawi.com
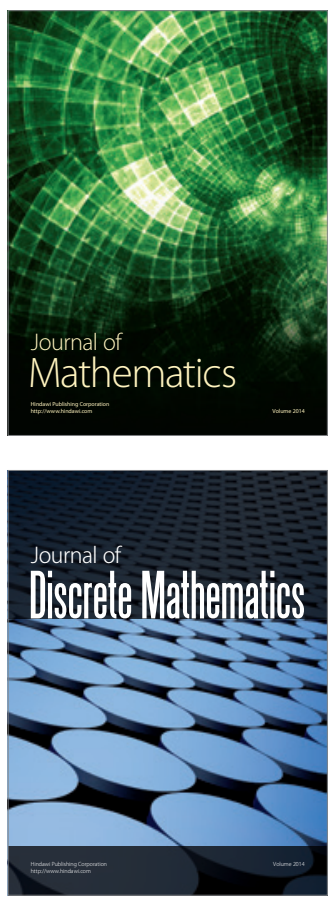

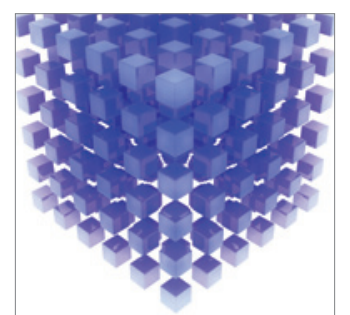

Mathematical Problems in Engineering
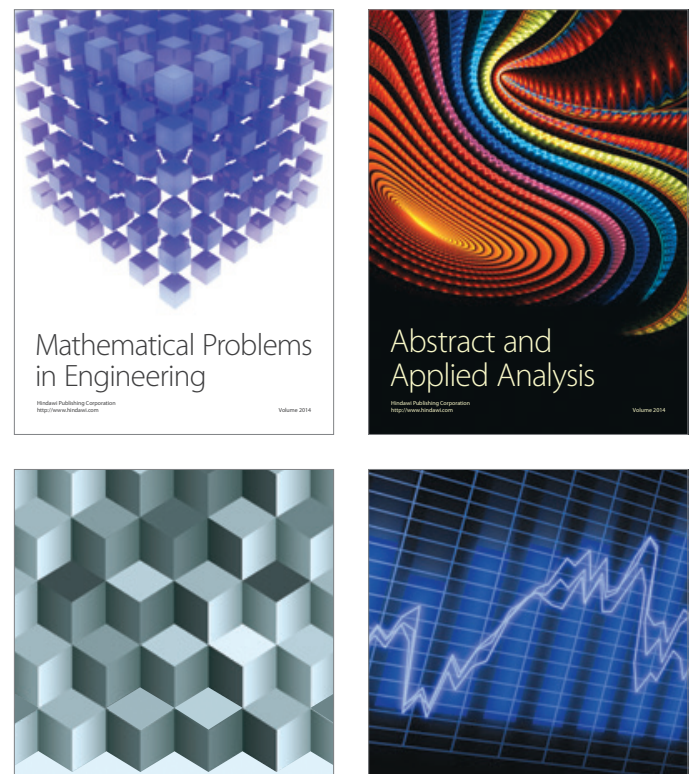

Journal of

Function Spaces

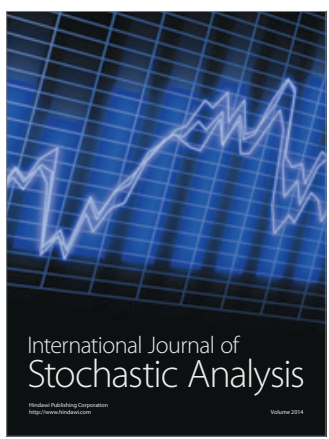

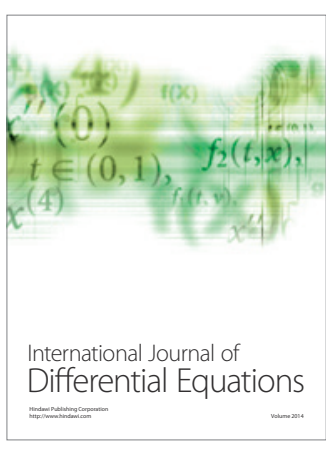
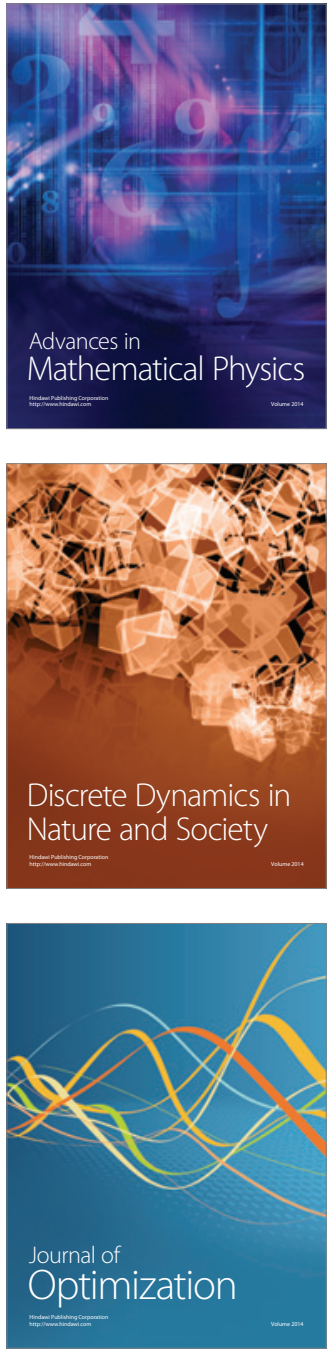